\title{
Confidence Region Approach for Assessing Bioequivalence and Biosimilarity Accounting for Heterogeneity of Variability
}

\author{
Jianghao Li and Shein-Chung Chow \\ School of Medicine, Duke University, Durham, NC 27705, USA \\ Correspondence should be addressed to Jianghao Li; davidleeljh@outlook.com
}

Received 27 August 2015; Accepted 23 November 2015

Academic Editor: Steve Su

Copyright ( $92015 \mathrm{~J}$. Li and S.-C. Chow. This is an open access article distributed under the Creative Commons Attribution License, which permits unrestricted use, distribution, and reproduction in any medium, provided the original work is properly cited.

\begin{abstract}
For approval of generic drugs, the FDA requires that evidence of bioequivalence in average bioequivalence in terms of drug absorption be provided through the conduct of a bioequivalence study. A test product is said to be average bioequivalent to a reference (innovative) product if the $90 \%$ confidence interval of the ratio of means (after log-transformation) is totally within $(80 \%, 125 \%)$. This approach is considered a one-parameter approach, which does not account for possible heterogeneity of variability between drug products. In this paper, we study a two-parameter approach (i.e., confidence region approach) for assessing bioequivalence, which can also be applied to assessing biosimilarity of biosimilar products. The proposed confidence region approach is compared with the traditional one-parameter approach both theoretically and numerically (i.e., simulation study) for finite sample performance.
\end{abstract}

\section{Introduction}

For approval of generic drug products, the United States (U.S.) Food and Drug Administration (FDA) requires that evidence of average bioequivalence (ABE) in terms of drug absorption in blood stream be provided. The evidence can only be obtained through the conduct of a bioequivalence study. Two drug products are claimed to be average bioequivalent if the $90 \%$ confidence interval for the ratio of means of the two drug products (i.e., $\mu_{T} / \mu_{R}$, where $\mu_{T}$ and $\mu_{R}$ are means of the test product and the reference product, resp.) based on log-transformed data is totally within the bioequivalence limits of $(80 \%, 125 \%)$. We will refer to this approach as the one-parameter approach. One of the major criticisms of this approach is that it ignores possible heterogeneity of variability between the two drug products.

Alternatively, Chow and Shao [1] proposed considering a confidence region approach (which we will refer to as a twoparameter approach) for assessing average bioequivalence. In other words, we consider confidence region (ellipse) for $\left(\mu_{T}, \mu_{R}\right)$ (two-parameter approach) rather than confidence interval for $\mu_{T} / \mu_{R}$ (one-parameter approach). Hsu and $\mathrm{Lu}$ [2] studied the relationship between the one-parameter approach and the two-parameter approach. As indicated by Chow and
Shao and $\mathrm{Hsu}$ and $\mathrm{Lu}$, the one-parameter approach is a special case of the two-parameter approach. In this paper, the proposed confidence region method is compared with the one-parameter approach by comparing the slopes of the tangent lines that hit the confidence region (ellipse). In addition, the probability of consistency (i.e., the probability of claiming bioequivalence based on the proposed method given that $T$ and $R$ are claimed to be bioequivalent based on the traditional one-parameter approach) will be evaluated.

In the next section, the traditional one-parameter approach is briefly outlined. The proposed confidence region approach is described in Section 3. Section 4 compares the proposed method with the traditional one-parameter approach both theoretically and via simulation study for finite sample performance. Also included in this section is the evaluation of the probability of inconsistency. An example is presented to illustrate the proposed method in Section 5. Some concluding remarks are given in the last section.

\section{Confidence Interval Approach for Assessing Average Bioequivalence}

In practice, the classic (shortest) confidence interval (CI) of $\mu_{T} / \mu_{R}$ is usually derived using the one-parameter method. 
According to the 2003 FDA guidance [3], bioequivalence should be assessed based on the log-transformed data of primary pharmacokinetic (PK) parameters such as AUC (area under the blood concentration-time curves) and $C_{\max }$ (maximum concentration). This means the calculation of CI for $\mu_{T} / \mu_{R}$ can be obtained from the CI of $\log \mu_{T} / \mu_{R}=\log \mu_{T}-$ $\log \mu_{R}$. Assuming that the data follow a lognormal distribution, let $\bar{X}_{T}$ and $\bar{X}_{R}$ be the sample means for the test and reference products, respectively (after log-transformation); the classic $(1-2 \alpha) \times 100 \%$ confidence interval can then be obtained based on the following statistics:

$$
T=\frac{\left(\bar{X}_{T}-\bar{X}_{R}\right)-\left(\mu_{T}-\mu_{R}\right)}{S} \sqrt{\frac{n_{T} n_{R}\left(n_{T}+n_{R}-2\right)}{n_{T}+n_{R}}},
$$

where $n_{T}$ and $n_{R}$ are the numbers of subjects in the test and reference group, respectively. Define $S$ as $S^{2} \triangleq$ $\sum_{i}\left(X_{T i}-\bar{X}_{T}\right)^{2}+\sum_{i}\left(X_{R i}-\bar{X}_{R}\right)^{2}$, where $X_{T i}$ and $X_{R i}$ are the responses of subject $i$ who receives test formulation and reference product, respectively. Under the assumption of normality and homogeneity, it can be verified that $T$ follows a central $t$ distribution with $\left(n_{T}+n_{R}-2\right)$ degrees of freedom. Thus, the $90 \%$ confidence interval for $\log \mu_{T}-\log \mu_{R}=$ $\log \mu_{T} / \mu_{R}$ can be obtained,

$$
\begin{gathered}
L_{1}=\left(\bar{X}_{T}-\bar{X}_{R}\right)-Q S \sqrt{\frac{n_{T}+n_{R}}{n_{T} n_{R}\left(n_{T}+n_{R}-2\right)}}, \\
U_{1}=\left(\bar{X}_{T}-\bar{X}_{R}\right)+Q S \sqrt{\frac{n_{T}+n_{R}}{n_{T} n_{R}\left(n_{T}+n_{R}-2\right)}},
\end{gathered}
$$

where $Q$ is the 95th quantile of a central $t$ distribution with $\left(n_{T}+n_{R}-2\right)$ degrees of freedom. The exact confidence interval for $\mu_{T} / \mu_{R}$ can thus be obtained by taking exponential of $L_{1}$ and $U_{1}$ as follows:

$$
\begin{aligned}
& L_{2}=\exp \left(\left(\bar{X}_{T}-\bar{X}_{R}\right)-Q S \sqrt{\frac{n_{T}+n_{R}}{n_{T} n_{R}\left(n_{T}+n_{R}-2\right)}}\right), \\
& U_{2}=\exp \left(\left(\bar{X}_{T}-\bar{X}_{R}\right)+Q S \sqrt{\frac{n_{T}+n_{R}}{n_{T} n_{R}\left(n_{T}+n_{R}-2\right)}}\right) .
\end{aligned}
$$

Let $L$ and $U$ be the respective lower and upper bioequivalence limits. Then average bioequivalence is claimed if

$$
\left(L_{2}, U_{2}\right) \in(L, U) \text {, }
$$

or

$$
\left(L_{1}, U_{1}\right) \in(\log L, \log U) .
$$

Meanwhile, we could also derive the criteria of confidence interval approach based on raw data. Assuming that $\bar{X}_{R}=\mu_{R}$, $n_{T}=n_{R}=n$, and we could get

$$
\begin{aligned}
& \frac{\bar{X}_{T}}{\bar{X}_{R}}-\frac{Q S}{\bar{X}_{R}} \sqrt{\frac{1}{n(n-1)}}>L, \\
& \frac{\bar{X}_{T}}{\bar{X}_{R}}+\frac{Q S}{\bar{X}_{R}} \sqrt{\frac{1}{n(n-1)}}<U .
\end{aligned}
$$

\section{Confidence Region Approach for Assessing Bioequivalence and Biosimilarity}

Chow and Shao [1] proposed a confidence region approach in assessing average bioequivalence. They performed bioequivalence testing under a crossover design and derived a confidence region for $\left(\mu_{T}, \mu_{R}\right)$ based on the raw data. Their method is similar to statistics derived in the third case below. In what follows, we will further expand Chow and Shao's notion more specifically working on the raw data and then discuss the relationship between using raw data and logtransformed data. We will mainly focus on the results in parallel design and crossover design whose period effects and sequence effects have been ruled out.

We will first look into the cases of raw data before eventually moving on to log-transformed data in Scenario 6. From Scenarios 1 to 5 , we suppose that $n_{T}$ subjects receive the test product $(T)$ and $n_{R}$ subjects receive the reference product $(R)$. Assume that the response of $T\left(X_{T}\right)$ follows a normal distribution, that is, $X_{T} \sim N\left(\mu_{T}, \sigma_{T}{ }^{2}\right)$, and the response of $R$ also follows a normal distribution, that is, $X_{R} \sim N\left(\mu_{R}, \sigma_{R}^{2}\right)$. Denote by $\bar{X}_{T}$ and $\bar{X}_{R}$ the sample mean responses of $T$ and $R$, respectively. We will consider the following possible scenarios.

Scenario $1\left(X_{T}\right.$ and $X_{R}$ are independent with $\sigma_{T}$ and $\sigma_{R}$ known $). X_{T i}\left(i=1,2, \ldots, n_{T}\right)$ and $X_{R i}\left(i=1,2, \ldots, n_{R}\right)$ are independent and identically distributed (i.i.d.); that is, $\bar{X}_{T} \sim N\left(\mu_{T}, \sigma_{T}{ }^{2} / n_{T}\right), \bar{X}_{R} \sim N\left(\mu_{R}, \sigma_{R}{ }^{2} / n_{R}\right)$. Thus, we have

$$
\begin{aligned}
\bar{X} \triangleq\left(\begin{array}{l}
\bar{X}_{T} \\
\bar{X}_{R}
\end{array}\right) \sim N(\mu, \Sigma), & \\
\mu & =\left(\begin{array}{l}
\mu_{T} \\
\mu_{R}
\end{array}\right), \Sigma=\left(\begin{array}{cc}
\frac{\sigma_{T}{ }^{2}}{n_{T}} & 0 \\
0 & \frac{\sigma_{R}^{2}}{n_{R}}
\end{array}\right) .
\end{aligned}
$$

It can be verified that $(\bar{X}-\mu)^{\prime} \Sigma^{-1}(\bar{X}-\mu)$ follows a chi-square distribution with 2 degrees of freedom. Suppose $q$ is the $(1-$ $\alpha)$ th quantile of the distribution. Then $(\bar{X}-\mu)^{\prime} \Sigma^{-1}(\bar{X}-\mu) \leq q$ is the $(1-\alpha) \times 100 \%$ confidence region for $\mu$. Thus, we have

$$
\frac{\left(\mu_{T}-\bar{X}_{T}\right)^{2}}{q \sigma_{T}^{2} / n_{T}}+\frac{\left(\mu_{R}-\bar{X}_{R}\right)^{2}}{q \sigma_{R}^{2} / n_{R}} \leq 1 .
$$

In order to compare with the one-parameter approach, that is, $90 \%$ confidence interval of $\mu_{T} / \mu_{R}$ is totally within ( $80 \%$, $125 \%$ ) after log-transformation, consider the tangent line with the form of $\mu_{T}=k \mu_{R}$ intercepting the ellipse. With some calculation, it can be verified that 


$$
k=\frac{\bar{X}_{T} \bar{X}_{R} \pm q \sigma_{T} \sigma_{R} \sqrt{\left(\bar{X}_{T}^{2} /\left(q \sigma_{T}^{2} / n_{T}\right)+\bar{X}_{R}^{2} /\left(q \sigma_{R}{ }^{2} / n_{R}\right)-1\right)\left(1 / n_{R} n_{T}\right)}}{\bar{X}_{R}{ }^{2}-q \sigma_{R}{ }^{2} / n_{R}} .
$$

That gives the upper bound and lower bound of $\mu_{T} / \mu_{R}$. Bioequivalence is concluded if $k$ falls within the given bioequivalence limits $(L, U)$. Besides, the center of the ellipse $\left(\bar{X}_{T}, \bar{X}_{T}\right)$ should be within $y=L x$ and $y=U x$. With some calculation (the details are shown in the Appendix), the criteria are the same as the following:

$$
\begin{aligned}
\left(L \bar{X}_{R}-\bar{X}_{T}\right)^{2}> & \frac{q \sigma_{T}{ }^{2}}{n_{T}}+\frac{q \sigma_{R}{ }^{2}}{n_{R}} L^{2}, \\
\left(U \bar{X}_{R}-\bar{X}_{T}\right)^{2}> & \frac{q \sigma_{T}{ }^{2}}{n_{T}}+\frac{q \sigma_{R}{ }^{2}}{n_{R}} U^{2}, \\
L & <\frac{\bar{X}_{T}}{\bar{X}_{R}}<U .
\end{aligned}
$$

Figure 1 shows the criteria for bioequivalence of the confidence region method. The red lines are $\mu_{T}=L \mu_{R}$ and $\mu_{T}=$ $U \mu_{R}$, which are the lower and upper bound of the tangent lines. Bioequivalence is concluded only when the confidence region (the ellipse) is completely within the boundaries. That is, the center of the ellipse is within the area bounded by the two red lines while the slope of the tangent lines is within $[L, U]$. As is shown in Figure 1, the two red lines indicate $\mu_{T}=$ $L \mu_{R}$ and $\mu_{T}=U \mu_{R}$, respectively. The dotted lines indicate two tangent lines of the central ellipse that pass through the origin. Unlike the other two ellipses, which intersect with at least one of the two red lines, the central ellipse is right inside the area bordered by the red lines. Bioequivalence can only be claimed in this situation.

In order to calculate the possibility of inconsistency, we need to evaluate the distribution of relevant statistics. As $L$ and $U$ are constants, that is, $L=80 \%$ and $U=125 \%$, respectively, we could evaluate two statistics $\left(L \bar{X}_{R}-\bar{X}_{T}\right)$ and $\left(U \bar{X}_{R}-\bar{X}_{T}\right)$. It can be verified that both statistics follow normal distributions $\left(L \bar{X}_{R}-\bar{X}_{T}\right) \sim N\left(L \mu_{R}-\right.$ $\left.\mu_{T}, \sigma_{T}{ }^{2} / n_{T}+\left(\sigma_{R}{ }^{2} / n_{R}\right) L^{2}\right),\left(U \bar{X}_{R}-\bar{X}_{T}\right) \sim N\left(U \mu_{R}-\mu_{T}, \sigma_{T}{ }^{2} / n_{T}+\right.$ $\left.\left(\sigma_{R}^{2} / n_{R}\right) U^{2}\right)$. This is useful in the next section.

If we want to use mean difference $\left(\mu_{T}-\mu_{R}\right)$ to test bioequivalence, we can use the test statistics described in Scenario 6.

Scenario $2\left(X_{T}\right.$ and $X_{R}$ are dependent with known covariance matrix). Suppose the test product and the reference product have the same sample size and they have pairwise correlation; that is, suppose the correlation coefficient of $X_{T i}$ and $X_{R i}$ is $\rho$; then $\operatorname{cov}\left(\bar{X}_{T}, \bar{X}_{R}\right)=\rho \sigma_{T} \sigma_{R} / n$. Thus, we have

$$
\begin{aligned}
\bar{X} \triangleq\left(\begin{array}{l}
\bar{X}_{T} \\
\bar{X}_{R}
\end{array}\right) \sim N(\mu, \Sigma), & \\
& \mu=\left(\begin{array}{l}
\mu_{T} \\
\mu_{R}
\end{array}\right), \Sigma=\left(\begin{array}{cc}
\frac{\sigma_{T}^{2}}{n} & \frac{\rho \sigma_{T} \sigma_{R}}{n} \\
\frac{\rho \sigma_{T} \sigma_{R}}{n} & \frac{\sigma_{R}^{2}}{n}
\end{array}\right) .
\end{aligned}
$$

Similarly, it can be verified that $(\bar{X}-\mu)^{\prime} \Sigma^{-1}(\bar{X}-\mu)$ follows a chi-square distribution with 2 degrees of freedom and the $(1-\alpha) \times 100 \%$ confidence region is given by $(\bar{X}-\mu)^{\prime} \Sigma^{-1}(\bar{X}-$ $\mu) \leq q$. This leads to

$$
\begin{aligned}
& \frac{\left(\mu_{T}-\bar{X}_{T}\right)^{2}}{q \sigma_{T}^{2} / n}+\frac{\left(\mu_{R}-\bar{X}_{R}\right)^{2}}{q \sigma_{R}^{2} / n} \\
& -2 \rho \frac{\left(\mu_{R}-\bar{X}_{R}\right)\left(\mu_{T}-\bar{X}_{T}\right)}{q \sigma_{T} \sigma_{R} / n} \leq 1-\rho^{2} n^{2}, \\
& \Delta \triangleq\left[-\bar{X}_{T} \bar{X}_{R}+\frac{\left(\rho q \sigma_{T} \sigma_{R}\right)}{n}\right]^{2} \\
& -\left(\bar{X}_{R}^{2}-\frac{q \sigma_{R}^{2}}{n}\right)\left(\bar{X}_{T}^{2}-\frac{q \sigma_{T}^{2}}{n}\right), \\
& k=\frac{\bar{X}_{R} \bar{X}_{T}-\left(\rho q \sigma_{T} \sigma_{R}\right) / n \pm \sqrt{\Delta}}{\left(\bar{X}_{R}^{2}-\left(q \sigma_{R}^{2} / n\right)\right)} .
\end{aligned}
$$

Similarly, bioequivalence is concluded if $k$ is within $(L, U)$. That is,

$$
\begin{aligned}
\left(L \bar{X}_{R}-\bar{X}_{T}\right)^{2} & >\frac{q \sigma_{T}^{2}}{n}+\frac{q \sigma_{R}^{2}}{n} L^{2}-2 \frac{\rho q \sigma_{T} \sigma_{R} L}{n}, \\
\left(U \bar{X}_{R}-\bar{X}_{T}\right)^{2} & >\frac{q \sigma_{T}^{2}}{n}+\frac{q \sigma_{R}^{2}}{n} U^{2}-2 \frac{\rho q \sigma_{T} \sigma_{R} L}{n}, \\
L & <\frac{\bar{X}_{T}}{\bar{X}_{R}}<U .
\end{aligned}
$$

In this case, $\left(L \bar{X}_{R}-\bar{X}_{T}\right) \sim N\left(\mu_{T}-y \mu_{R}, \sigma_{T}{ }^{2} / n+\left(\sigma_{R}{ }^{2} / n\right) L^{2}-\right.$ $\left.2\left(\rho \sigma_{T} \sigma_{R} L / n\right)\right),\left(R \bar{X}_{R}-\bar{X}_{T}\right) \sim N\left(\mu_{T}-y \mu_{R}, \sigma_{T}^{2} / n+\left(\sigma_{R}{ }^{2} / n\right) R^{2}-\right.$ $\left.2\left(\rho q \sigma_{T} \sigma_{R} L / n\right)\right)$.

Scenario 3 (covariance matrix is unknown). In this case, the covariance could only be replaced by its unbiased and 


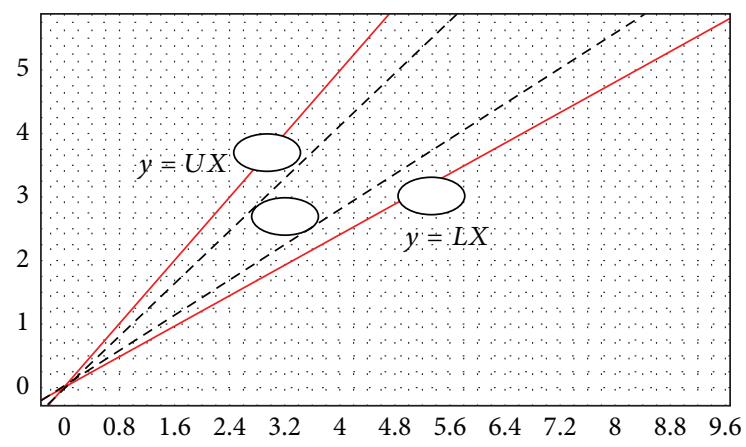

FiguRE 1: Bioequivalence criteria for two-parameter approach.

consistent estimate. For simplicity, suppose $n_{T}=n_{R}=n$; that is, the responses $X_{T i}$ and $X_{R i}(i=1,2, \ldots, n)$ can be expressed as $X_{i} \triangleq\left(\begin{array}{c}X_{R i} \\ X_{T i}\end{array}\right) . A^{2} \triangleq \sum_{i}\left(X_{i}-\mu\right)\left(X_{i}-\mu\right)^{\prime}$ is the deviation matrix. Intuitively $(1 /(n-1)) A$ is an unbiased and consistent estimate. Thus the following test statistic $T^{2} \triangleq n(\bar{X}-$ $\mu)^{\prime}((1 /(n-1)) A)^{-1}(\bar{X}-\mu)$ follows a Hotelling distribution with dimension equal to 2 and degree of freedom equal to $n-1$. Thus it can be shown that $((n-1+1-2) / 2(n-1)) T^{2} \sim$ $F(2, n-2)$. Similarly the $(1-\alpha) \times 100 \%$ confidence region for $\mu$ can be obtained as

$$
\frac{n(n-2)}{2}(\bar{X}-\mu)^{\prime} A^{-1}(\bar{X}-\mu) \leq q,
$$

where $q$ is the $(1-\alpha)$ th quantile of $F(2, n-2)$. As $A$ is symmetric, we assume its form as $\left(\begin{array}{cc}a^{2} & \rho a b \\ \rho a b & b^{2}\end{array}\right)$. Thus, after transformation the confidence region can be obtained as

$$
\begin{aligned}
& \frac{\left(\mu_{T}-\bar{X}_{T}\right)^{2}}{q b^{2}}+\frac{\left(\mu_{R}-\bar{X}_{R}\right)^{2}}{q a^{2}} \\
& \quad-2 \rho \frac{\left(\mu_{R}-\bar{X}_{T}\right)\left(\mu_{T}-\bar{X}_{T}\right)}{q a b} \leq \frac{2\left(1-\rho^{2}\right)}{n(n-2)} .
\end{aligned}
$$

Suppose the tangent line is $\mu_{T}=k \mu_{R}$; solving the above equation, $k$ can be obtained as follows:

$$
\begin{aligned}
\Delta \triangleq & {\left[-\bar{X}_{R} \bar{X}_{T}+\left(\frac{2 \rho a b q}{n(n-2)}\right)\right]^{2} } \\
& -\left(\bar{X}_{R}^{2}-\frac{2 a^{2} q}{n(n-2)}\right)\left(\bar{X}_{T}^{2}-\frac{2 b^{2} q}{n(n-2)}\right), \\
k= & \frac{\bar{X}_{R} \bar{X}_{T}-\left(2 \rho a^{2} b^{2} q / n(n-2)\right) \pm \sqrt{\Delta}}{\left(\bar{X}_{R}^{2}-2 a^{2} q / n(n-2)\right)} .
\end{aligned}
$$

Bioequivalence is claimed when $k$ is within $(L, U)$; that is,

$$
\begin{aligned}
\left(L \bar{X}_{R}-\bar{X}_{T}\right)^{2}> & \frac{2 q b^{2}}{n(n-2)}+\frac{2 q a^{2}}{n(n-2)} L^{2} \\
& -4 \frac{\rho a b q}{n(n-2)} L, \\
\left(U \bar{X}_{R}-\bar{X}_{T}\right)^{2}> & \frac{2 q b^{2}}{n(n-2)}+\frac{2 q a^{2}}{n(n-2)} U^{2} \\
& -4 \frac{\rho a b q}{n(n-2)} U, \\
L< & \frac{\bar{X}_{T}}{\bar{X}_{R}}<U .
\end{aligned}
$$

Scenario 4 (variance comparison). As most biosimilar products are highly variable, it is crucial to compare the variances of test and reference products. Comparing variabilities using the one-parameter approach has been studied in the literature. See, for example, Pitman; Morgan; Chow and Tse; Lee et al. [4-7]. Comparing variabilities using the two-parameter approach is also feasible, which is briefly described below.

As an example, consider the problem of testing homogeneity. In variances, suppose $X$ is a $p \times 1$ vector that contains $n$ responses for all the reference and test products and $X \sim$ $N(\mu, \Sigma)$. The null hypothesis of interest is that $H_{0}: \Sigma=I_{p}$, where $I_{p}$ is a $p \times p$ identity matrix (the matrix with ones on the main diagonal and zeros elsewhere). Define $A \triangleq \sum_{i}\left(X_{i}-\right.$ $\mu)\left(X_{i}-\mu\right)^{\prime}$ and $\lambda=\exp (-(1 / 2) \operatorname{tr}(A))|A|^{n / 2}(e / n)^{n p / 2}$. Define $\varepsilon=-2 \ln (\lambda)$. Then it can be verified that $\varepsilon$ approximately follows chi-square distribution with the degree of freedom of $p(p+1) / 2$.

In more general cases, we could test multiple reference products with multiple test products at the same time. In such cases, $H_{0}: \Sigma=\Sigma_{0}$, where $\Sigma_{0}$ may not be an identity matrix as the variance of different reference products need not be the same. Then defining

$$
\lambda^{\prime}=\exp \left(-\frac{1}{2} \operatorname{tr}\left(A \Sigma_{0}{ }^{-1}\right)\right)\left|A \Sigma_{0}{ }^{-1}\right|^{n / 2}\left(\frac{e}{n}\right)^{n p / 2},
$$

it can be proved that $-2 \ln \left(\lambda^{\prime}\right)$ also follows $\chi^{2}(p(p+1) / 2)$.

Scenario 5 (test for biosimilar products). As indicated in Chow and Liu [8], biosimilar products can be very variable due to their susceptibility to environment conditions such as light and temperature. So they are not identical to reference products. Following similar idea, the biosimilarity of biosimilar products can be assessed using the two-parameter approach by taking the possible variation into account. In other words, it can be tested whether

$$
L \mu_{R}+d<\mu_{T}<U \mu_{R}+e
$$

holds true, where $m$ and $n$ are intercepts that may not be zeros. Note that these may not exist; this case may not be an easy solution with the one-parameter approach. However, we can formulate the criteria for testing bioequivalence as follows. 


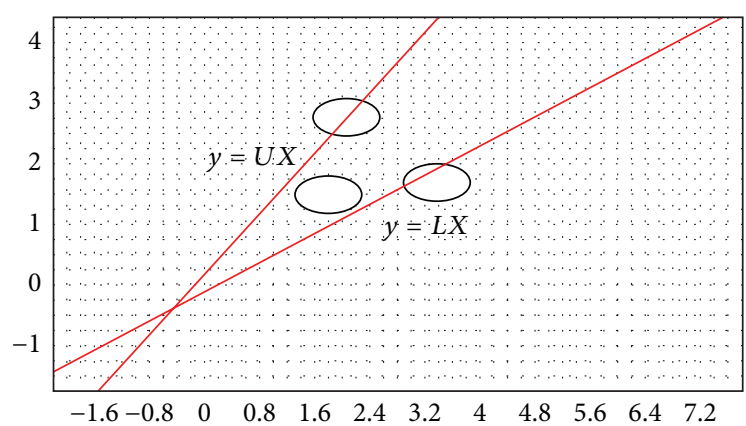

Figure 2: Biosimilarity criteria for two-parameter approach. we have

Adopting the notations in Scenario 3 as described above,

$$
\frac{n(n-2)}{2}(\bar{X}-\mu)^{\prime} A^{-1}(\bar{X}-\mu) \leq q
$$

as the confidence region for $\left(\mu_{R}, \mu_{T}\right)$. Bioequivalence is claimed when this ellipse region is within the area bounded by $\mu_{T}=U \mu_{R}+e$ and $\mu_{T}=L \mu_{R}+d$. With some calculation, we could formulate the calculation as follows:

$$
\begin{aligned}
\left(L \bar{X}_{R}-\bar{X}_{T}+d\right)^{2}> & \frac{2 q b^{2}}{n(n-2)}+\frac{2 q a^{2}}{n(n-2)} L^{2} \\
& -4 \frac{\rho a b q}{n(n-2)} L, \\
\left(U \bar{X}_{R}-\bar{X}_{T}+e\right)^{2}> & \frac{2 q b^{2}}{n(n-2)}+\frac{2 q a^{2}}{n(n-2)} U^{2} \\
& -4 \frac{\rho a b q}{n(n-2)} U, \\
L \bar{X}_{R}+d< & \bar{X}_{T}<U \bar{X}_{R}+e,
\end{aligned}
$$

which is of approximately the same form as what have been derived in Scenario 3.

Figure 2 shows the criteria for biosimilarity. It is similar to Figure 1, but both lines in Figure 2 may not pass through the origin.

Scenario 6 (test based on log-transformed data). According to the 2003 FDA guidance [3], bioequivalence testing should be conducted based on the log-transformed data. Thus, the assumptions above should be modified so that the response (after log-transformation) of $T\left(X_{T}\right)$ follows $X_{T} \sim N\left(\log \left(\mu_{T}\right), \sigma_{T}^{2}\right)$ and the response of $R$ follows $X_{R} \sim$ $N\left(\log \left(\mu_{R}\right), \sigma_{R}^{2}\right)$. The bioequivalence criterion is

$$
L<\frac{\mu_{T}}{\mu_{R}}<U
$$

This indicates that

$$
\log \left(\mu_{R}\right)+\log L<\log \left(\mu_{T}\right)<\log \left(\mu_{R}\right)+\log U .
$$

Note that this is simply a special case for what was derived in Scenario 5 by making the slopes of both lines equal 1 . The equation then becomes

$$
\begin{aligned}
\left(\bar{X}_{R}-\bar{X}_{T}+\log L\right)^{2}> & \frac{2 q b^{2}}{n(n-2)}+\frac{2 q a^{2}}{n(n-2)} \\
& -4 \frac{\rho a b q}{n(n-2)}, \\
\left(\bar{X}_{R}-\bar{X}_{T}+\log U\right)^{2}> & \frac{2 q b^{2}}{n(n-2)}+\frac{2 q a^{2}}{n(n-2)} \\
& -4 \frac{\rho a b q}{n(n-2)},
\end{aligned}
$$$$
\log L<\bar{X}_{T}-\bar{X}_{R}<\log U \text {. }
$$

\section{Comparison between One-Parameter Approach and Two-Parameter Approach}

In this section, we will compare the proposed method with the traditional one-parameter method. For simplicity, assume that $n_{T}=n_{R}=n$, and we only need to be concerned with the case where covariance matrix is unknown in the twoparameter approach. According to the results is Section 2, the criteria for bioequivalence in one-parameter approach are equivalent to

$$
\begin{gathered}
\bar{X}_{T}-L \bar{X}_{R}>\sqrt{Q^{2} S^{2} \frac{1}{n(n-1)}}, \\
U \bar{X}_{R}-\bar{X}_{T}>\sqrt{Q^{2} S^{2} \frac{1}{n(n-1)}} .
\end{gathered}
$$

Similarly, in the two-parameter approach, bioequivalence criteria are equivalent to the following form:

$$
\begin{aligned}
& \bar{X}_{T}-L \bar{X}_{R} \\
& \quad>\sqrt{\frac{2 q b^{2}}{n(n-2)}+\frac{2 q a^{2}}{n(n-2)} L^{2}-4 \frac{\rho a b q}{n(n-2)} L,} \\
& U \bar{X}_{R}-\bar{X}_{T} \\
& >\sqrt{\frac{2 q b^{2}}{n(n-2)}+\frac{2 q a^{2}}{n(n-2)} U^{2}-4 \frac{\rho a b q}{n(n-2)} U .}
\end{aligned}
$$

Compared with the two-parameter approach, it is obvious that $S^{2}=a^{2}+b^{2}$. Given that $T$ and $R$ are claimed to be bioequivalent based on one-parameter approach, then we could suppose that ${\sigma_{T}}^{2}={\sigma_{R}}^{2}=\sigma^{2}$ due to the homogeneity 


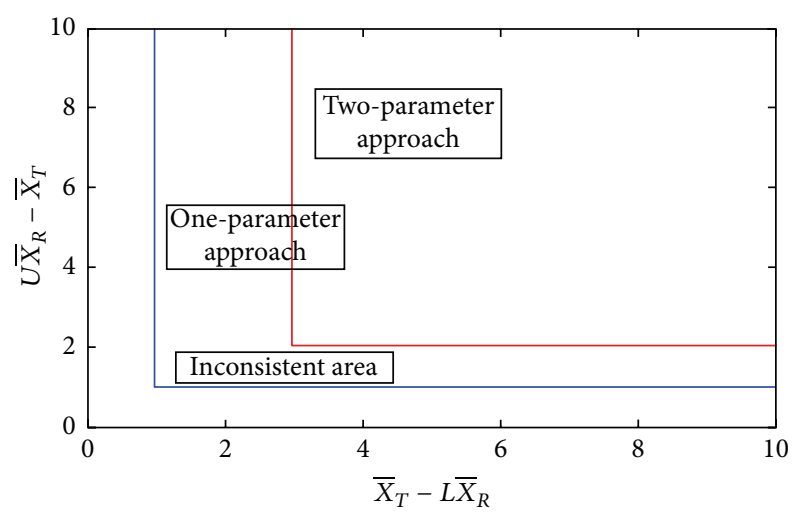

Figure 3: Comparison of two approaches' criteria.

assumption in the one-parameter model. Then $\left(L \bar{X}_{R}-\bar{X}_{T}\right) \sim$ $N\left(L \mu_{R}-\mu_{T},\left(\left(1+L^{2}\right) / n\right) \sigma^{2}\right),\left(U \bar{X}_{R}-\bar{X}_{T}\right) \sim N\left(U \mu_{R}-\mu_{T},((1+\right.$ $\left.\left.\left.U^{2}\right) / n\right) \sigma^{2}\right)$. So $\left(U \bar{X}_{R}-\bar{X}_{T}, \bar{X}_{T}-L \bar{X}_{R}\right)^{\prime} \sim N(\mu, \Sigma)$, where

$$
\begin{aligned}
& \mu=\left(\mu_{1}, \mu_{2}\right)^{\prime}=\left(U \mu_{R}-\mu_{T}, \mu_{T}-L \mu_{R}\right)^{\prime}, \\
& \Sigma=\left(\begin{array}{cc}
\frac{\left(1+U^{2}\right)}{n} \sigma^{2} & -\frac{1}{n} \sigma^{2}-\frac{U^{2} L^{2}}{n} \sigma^{2} \\
-\frac{1}{n} \sigma^{2}-\frac{U^{2} L^{2}}{n} \sigma^{2} & \frac{\left(1+L^{2}\right)}{n} \sigma^{2}
\end{array}\right) .
\end{aligned}
$$

Figure 3 shows the relationship of the two approaches. The area in the upper right position of the red lines is the bioequivalent area of the two-parameter approach, while the upper right area of the blue lines is that of the one-parameter approach. The two approaches are inconsistent; that is, bioequivalence cannot be concluded by the two-parameter approach given the fact that $T$ and $R$ are bioequivalent based on the one-parameter approach, if the vector $\left(U \bar{X}_{R}-\bar{X}_{T}, \bar{X}_{T}-\right.$ $L \bar{X}_{R}$ ) falls into the area between the red line and the blue line. Defining the area between the red lines and blue lines as inconsistency area, we can calculate the possibility of the random vector not falling into this region.

We will briefly compare these two approaches. On the one hand, when $\bar{X}_{T}$ and $\bar{X}_{R}$ vary greatly, for example, $\bar{X}_{T}$ is 1.5 times bigger than $\bar{X}_{R}$, bioequivalence would not be claimed under either approach, as $U \bar{X}_{R}-\bar{X}_{T}$ is smaller than zero. Thus both approaches are rigorous in the sense that bioequivalence can only be claimed when $\bar{X}_{T}$ and $\bar{X}_{R}$ are sufficiently close. On the other hand, when $\bar{X}_{T}$ and $\bar{X}_{R}$ are close, the influence of bigger variation differs in two approaches. Noting that $Q^{2}<2 q$, it can be easily estimated that when $\rho$ is relatively small, $\sqrt{2 q b^{2} / n(n-2)+\left(2 q a^{2} / n(n-2)\right) L^{2}-4(\rho a b q / n(n-2)) L}>$ $\sqrt{Q^{2} S^{2}(1 / n(n-1))}$. That means the two-parameter approach is more rigorous than the one-parameter approach. But when $\rho$ is rather big, the situation is more sophisticated, as it is difficult to tell which method is more conservative. Thus, we try to delineate the relationship of the two approaches by calculating the following conditional probabilities.
Suppose $P_{1}=P$ (two-parameter approach claims bioequivalence $\mid$ one-parameter approach claims bioequivalence), and $\Phi(x)$ is the cumulative distribution function of standard normal distribution; then we could get the possibility of $\left(U \bar{X}_{R}-\bar{X}_{T}, \bar{X}_{T}-L \bar{X}_{R}\right)^{\prime}$ falling into the inconsistency area $P_{1 U L}:$

$$
\begin{aligned}
& P_{1 U L}=\iint_{(y, x) \in I_{1}} \frac{1}{2 \pi|\Sigma|^{-1 / 2}} \exp \left[-\frac{1}{2}\left(x-\mu_{1}, y-\mu_{2}\right)^{\prime}\right. \\
& \left.\cdot \Sigma^{-1}\left(x-\mu_{1}, y-\mu_{2}\right)\right] \\
& \quad+\iint_{(y, x) \in I_{2}} \frac{1}{2 \pi|\Sigma|^{-1 / 2}} \exp \left[-\frac{1}{2}\left(x-\mu_{1}, y-\mu_{2}\right)^{\prime}\right. \\
& \left.\cdot \Sigma^{-1}\left(x-\mu_{1}, y-\mu_{2}\right)\right],
\end{aligned}
$$

where

$$
\begin{aligned}
I_{1} & =\left\{(y, x) \mid y=\bar{X}_{T}-L \bar{X}_{R}, x=U \bar{X}_{R}-\bar{X}_{T}, \quad y\right. \\
& \in\left(\sqrt{Q^{2} S^{2} \frac{1}{n(n-1)}},\right. \\
& \sqrt{\frac{2 q b^{2}}{n(n-2)}+\frac{2 q a^{2}}{n(n-2)} L^{2}-4 \frac{\rho a b q}{n(n-2)} L}, x \\
& \left.\in\left(\sqrt{Q^{2} S^{2} \frac{1}{n(n-1)}},+\infty\right)\right\}, \\
I_{2} & =\left\{(y, x) \mid y=\bar{X}_{T}-L \bar{X}_{R}, x=U \bar{X}_{R}-\bar{X}_{T}, y\right. \\
& \in\left(\sqrt{\frac{2 q b^{2}}{n(n-2)}+\frac{2 q a^{2}}{n(n-2)} L^{2}-4 \frac{\rho a b q}{n(n-2)}} L,\right. \\
& +\infty), x \in\left(\sqrt{Q^{2} S^{2} \frac{1}{n(n-1)}},\right. \\
& \left.\left.\sqrt{\frac{2 q b^{2}}{n(n-2)}+\frac{2 q a^{2}}{n(n-2)} U^{2}-4 \frac{\rho a b q}{n(n-2)} U}\right)\right\} .
\end{aligned}
$$

Thus the possibility of consistency equals $P_{1}=1-P_{1 U L}$.

On the other hand, given the fact that two products are claimed to be bioequivalent under the two-parameter approach, then we could not assume homogeneity in this model. So $\left(U \bar{X}_{R}-\bar{X}_{T}, \bar{X}_{T}-L \bar{X}_{R}\right)^{\prime} \sim N\left(\mu, \Sigma^{\prime}\right)$, where

$$
\Sigma^{*}=\left(\begin{array}{cc}
\frac{U^{2}}{n} \sigma_{R}^{2}+\frac{1}{n} \sigma_{T}^{2} & -\frac{1}{n} \sigma_{T}^{2}-\frac{U^{2} L^{2}}{n} \sigma_{R}^{2} \\
-\frac{1}{n} \sigma_{T}^{2}-\frac{U^{2} L^{2}}{n} \sigma_{R}^{2} & \frac{L^{2}}{n} \sigma_{R}{ }^{2}+\frac{1}{n} \sigma_{T}^{2}
\end{array}\right) .
$$


Assume $P_{2}=P$ (one-parameter approach claims bioequivalence | two-parameter approach claims bioequivalence); we could similarly derive the value of $P_{2}$ as follows:

$$
\begin{aligned}
& P_{2 U L}=\iint_{(y, x) \in I_{3}} \frac{1}{2 \pi\left|\Sigma^{*}\right|^{-1 / 2}} \exp \left[-\frac{1}{2}\left(x-\mu_{1}, y-\mu_{2}\right)^{\prime}\right. \\
& \left.\cdot \Sigma^{*^{-1}}\left(x-\mu_{1}, y-\mu_{2}\right)\right] \\
& =\iint_{(y, x) \in I_{4}} \frac{1}{2 \pi\left|\Sigma^{*}\right|^{-1 / 2}} \exp \left[-\frac{1}{2}\left(x-\mu_{1}, y-\mu_{2}\right)^{\prime}\right. \\
& \left.\cdot \Sigma^{*^{-1}}\left(x-\mu_{1}, y-\mu_{2}\right)\right]
\end{aligned}
$$

where

$$
\begin{aligned}
& I_{3}=\left\{(y, x) \mid y=\bar{X}_{T}-L \bar{X}_{R}, x=U \bar{X}_{R}-\bar{X}_{T}, y\right. \\
& \epsilon\left(\sqrt{\frac{2 q b^{2}}{n(n-2)}+\frac{2 q a^{2}}{n(n-2)} L^{2}-4 \frac{\rho a b q}{n(n-2)}} L,\right. \\
& \left.\sqrt{Q^{2} S^{2} \frac{1}{n(n-1)}}\right), x \\
& \epsilon\left(\sqrt{\frac{2 q b^{2}}{n(n-2)}+\frac{2 q a^{2}}{n(n-2)} U^{2}-4 \frac{\rho a b q}{n(n-2)} U},\right. \\
& +\infty)\} \\
& I_{4}=\left\{(y, x) \mid y=\bar{X}_{T}-L \bar{X}_{R}, x=U \bar{X}_{R}-\bar{X}_{T}, y\right. \\
& \in\left(\sqrt{Q^{2} S^{2} \frac{1}{n(n-1)}},+\infty\right), x \\
& \epsilon\left(\sqrt{\frac{2 q b^{2}}{n(n-2)}+\frac{2 q a^{2}}{n(n-2)} U^{2}-4 \frac{\rho a b q}{n(n-2)} U},\right. \\
& \left.\left.\sqrt{Q^{2} S^{2} \frac{1}{n(n-1)}}\right)\right\} .
\end{aligned}
$$

Thus,

$$
P_{2}=1-P_{2 U L}
$$

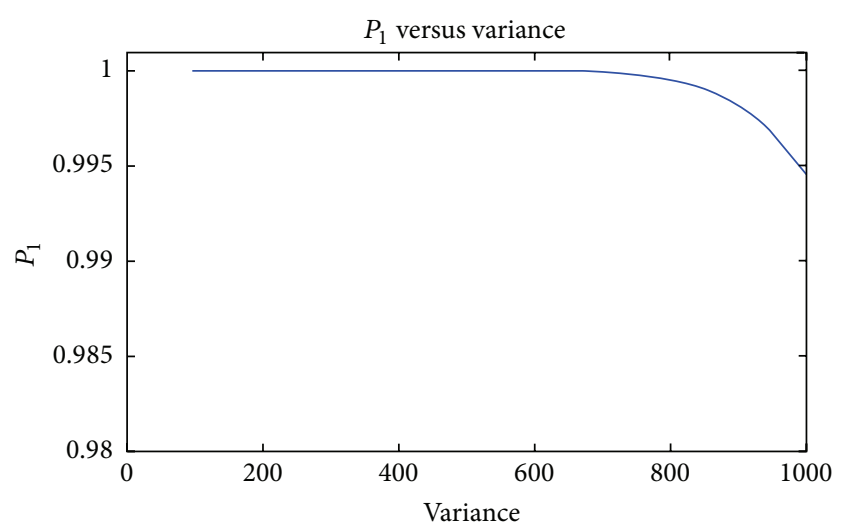

(a)

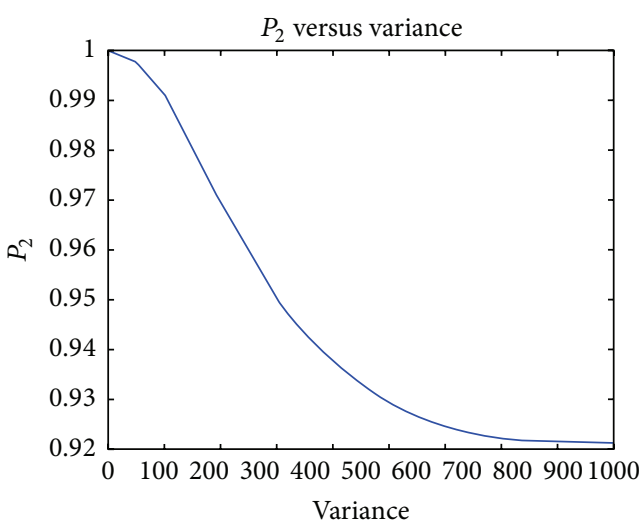

(b)

Figure 4: Changes of $P_{1}$ and $P_{2}$ against variance.

In order to evaluate the influence of variance on both approaches, we try to plot $P_{1}$ and $P_{2}$ against the variance. We set the sample means of reference and test products as constants. First, we suppose the variances of $T$ and $R$ are the same, that is, $\sigma_{R}{ }^{2}=\sigma_{T}{ }^{2}$, and change at the same time. The covariance coefficient $\rho$ equals 0.6 , referring to the example in the next section. We further suppose that the sample variances equal the actual variances of $T$ and $R$. We could calculate approximate values of $P_{1}$ and $P_{2}$ to draw the two plots shown in Figure 4.

From Figure 4, it can be seen that $P_{1}$ declined little when $\sigma_{R}{ }^{2}$ and $\sigma_{T}{ }^{2}$ change from 100 to 1000 , staying close to $100 \%$. But $P_{2}$ drops rather dramatically, which coincides with what was predicted earlier in this section. That is, the oneparameter approach tends to be more conservative when the variation becomes greater.

Besides, we are interested in what would happen when the variance of test products increases. This is an important question when it comes to assessment of biosimilarity. Thus we draw the plot shown in Figure 5.

In the calculation, $\sigma_{R}{ }^{2}$ is set as 400 (i.e., $\sigma_{R}=20$ ) and $\sigma_{T}{ }^{2}$ varies from 300 to 2500 . From Figure 5, we could see that $P_{2}$ has a single nadir and tends to increase when $\sigma_{T}{ }^{2}$ becomes way bigger than $\sigma_{T}{ }^{2}$. Eventually $P_{2}$ equals $100 \%$. This can be proved by noticing $2 q b^{2} / n(n-2)+\left(2 q a^{2} / n(n-2)\right) L^{2}-$ $4(\rho a b q / n(n-2)) L-Q^{2}\left(a^{2}+b^{2}\right)(1 / n(n-1))$ is a quadratic 


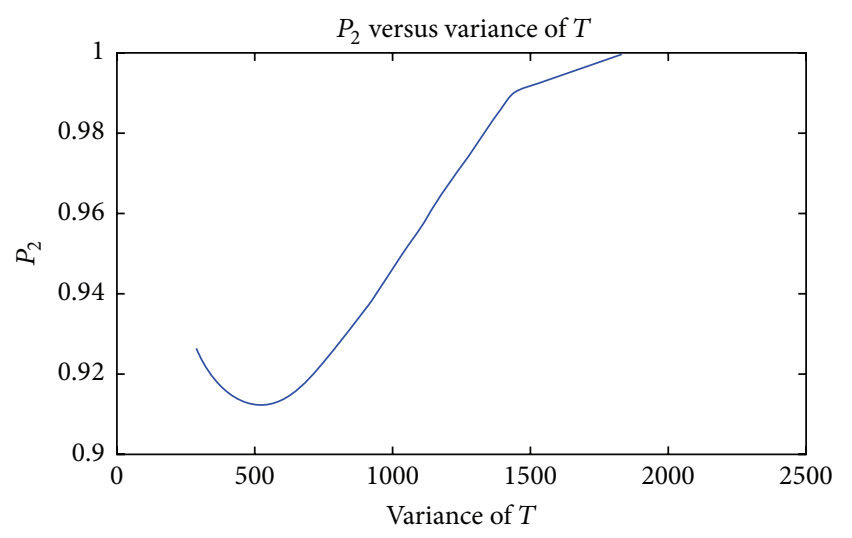

FIgure 5: Change of $P_{2}$ against ${\sigma_{T}}^{2}$.

function of $b$, with its quadratic coefficient bigger than zero. So the function value is above zero when $b$ is large enough. Thus $P_{2}$ turns $100 \%$ in that situation.

We could also discuss the relationship of two approaches based on log-transformed data. According to the results in Section 2 and Section 3 Scenario 6, the criteria for the
CI approach and proposed approach can be rewritten with simple transformation as

$$
\begin{aligned}
\bar{X}_{T}-\bar{X}_{R}> & \sqrt{Q^{2} S^{2} \frac{1}{n(n-1)}}+\log L, \\
\bar{X}_{T}-\bar{X}_{R}< & -\sqrt{Q^{2} S^{2} \frac{1}{n(n-1)}+\log U,} \\
\bar{X}_{T}-\bar{X}_{R}> & \sqrt{\frac{2 q b^{2}}{n(n-2)}+\frac{2 q a^{2}}{n(n-2)}-4 \frac{\rho a b q}{n(n-2)}} \\
& +\log L, \quad \\
\bar{X}_{T}-\bar{X}_{R}< & -\sqrt{\frac{2 q b^{2}}{n(n-2)}+\frac{2 q a^{2}}{n(n-2)}-4 \frac{\rho a b q}{n(n-2)}} \\
& +\log U,
\end{aligned}
$$

respectively. If bioequivalence or biosimilarity is claimed based on the one-parameter approach, then homoscedasticity is assumed; that is, $\sigma_{T}{ }^{2}=\sigma_{R}{ }^{2}=\sigma^{2}$. So $\left(\bar{X}_{T}-\bar{X}_{R}\right) \sim$ $N\left(\mu_{T}-\mu_{R},(2 / n) \sigma^{2}\right)$. The probability of inconsistency can be written as

$$
\begin{aligned}
P_{1 U L}^{\prime} & =\max \left\{\Phi\left(\frac{\left(\sqrt{2 q b^{2} / n(n-2)+2 q a^{2} / n(n-2)-4(\rho a b q / n(n-2))}+\log U\right)-\left(\mu_{T}-\mu_{R}\right)}{\sqrt{(2 / n) \sigma^{2}}}\right)\right. \\
& \left.-\Phi\left(\frac{\left(\sqrt{Q^{2} S^{2}(1 / n(n-1))}+\log U\right)-\left(\mu_{T}-\mu_{R}\right)}{\sqrt{(2 / n) \sigma^{2}}}\right), 0\right\}+\max \left\{\Phi\left(\frac{\left(\sqrt{Q^{2} S^{2}(1 / n(n-1))}+\log L\right)-\left(\mu_{T}-\mu_{R}\right)}{\sqrt{(2 / n) \sigma^{2}}}\right)\right. \\
& \left.-\Phi\left(\frac{\left(\sqrt{2 q b^{2} / n(n-2)+2 q a^{2} / n(n-2)-4(\rho a b q / n(n-2))}+\log L\right)-\left(\mu_{T}-\mu_{R}\right)}{\sqrt{(2 / n) \sigma^{2}}}\right), 0\right\} .
\end{aligned}
$$

Then $P_{1}=1-P_{1 U L}^{\prime}$.
Similarly, we could obtain $P_{2}$ based on log-transformed data. Under the hypothesis of heterogeneity, $\left(\bar{X}_{T}-\bar{X}_{R}\right) \sim$ $N\left(\mu_{T}-\mu_{R},(1 / n) \sigma_{T}^{2}+(1 / n) \sigma_{R}^{2}\right)$, thus

$$
\begin{aligned}
P_{2 U L}^{\prime} & =\max \left\{\Phi\left(\frac{\left(\sqrt{Q^{2} S^{2}(1 / n(n-1))}+\log U\right)-\left(\mu_{T}-\mu_{R}\right)}{\sqrt{(1 / n) \sigma_{T}^{2}+(1 / n) \sigma_{R}^{2}}}\right)\right. \\
& \left.-\Phi\left(\frac{\left(\sqrt{2 q b^{2} / n(n-2)+2 q a^{2} / n(n-2)-4(\rho a b q / n(n-2))}+\log U\right)-\left(\mu_{T}-\mu_{R}\right)}{\sqrt{(1 / n) \sigma_{T}^{2}+(1 / n) \sigma_{R}^{2}}}\right), 0\right\}
\end{aligned}
$$




$$
\begin{aligned}
& +\max \left\{\Phi\left(\frac{\left(\sqrt{2 q b^{2} / n(n-2)+2 q a^{2} / n(n-2)-4(\rho a b q / n(n-2))}+\log L\right)-\left(\mu_{T}-\mu_{R}\right)}{\sqrt{(1 / n) \sigma_{T}^{2}+(1 / n) \sigma_{R}^{2}}}\right)\right. \\
& \left.-\Phi\left(\frac{\left(\sqrt{Q^{2} S^{2}(1 / n(n-1))}+\log L\right)-\left(\mu_{T}-\mu_{R}\right)}{\sqrt{(1 / n) \sigma_{T}^{2}+(1 / n) \sigma_{R}^{2}}}\right), 0\right\} .
\end{aligned}
$$

Then $P_{2}=P_{2 U L}^{\prime}$.

In our simple simulation, we change ${\sigma_{T}}^{2}$ and $\sigma_{R}{ }^{2}$ from 10 to 100 and choose $n$ as 500 in order to ensure that bioequivalence can be claimed under both approaches. Then we found that $P_{2}$ constantly equals 1 while $P_{1}$ declines with the growth of variances, as is shown in Figure 6.

This means that, in cases where the confidence interval approach claims bioequivalence, our proposed approach is also likely to get to the same conclusion. But the twoparameter approach gives narrower confidence interval for $\bar{X}_{T}-\bar{X}_{R}$, so it provides greater power.

\section{An Example}

To illustrate the effects of the proposed approach, consider an example from Chow and Liu [8]. The study is a standard $2 \times$ 2 crossover design, conducted on 24 volunteers. Each subject would take either $50 \mathrm{mg}$ tablets (test formulation) or $5 \mathrm{~mL}$ of an oral suspension (reference formulation). AUC values were calculated with the blood samples obtained at different times. The data is given in Table 1.

It can be tested that there is no influence of period and sequence effects. When adopting the one-parameter approach, it can be calculated that $S=20202.89, \bar{X}_{T}=80.27$, $\bar{X}_{R}=82.56$, and $Q=1.68$; thus the three requirements can be satisfied in the one-parameter approach. On the other hand, $a^{2}=9948.28, b^{2}=10254.6, \rho a b q=6386.05$, and $q=2.56$, so it can also be verified that bioequivalence is concluded in the two-parameter approach.

We could also calculate the inconsistent probability in this example. Suppose the unbiased estimates of mean and variation are the accurate value. It is pretty hard to get the exact value by $P_{2 U L}$, but it should be smaller than

$$
\begin{aligned}
& P\left(\bar{X}_{T}-L \bar{X}_{R} \in\left(\sqrt{\frac{2 q b^{2}}{n(n-2)}+\frac{2 q a^{2}}{n(n-2)} L^{2}-4 \frac{\rho a b q}{n(n-2)}} L \sqrt{Q^{2} S^{2} \frac{1}{n(n-1)}}\right)\right) \\
& +P\left(U \bar{X}_{R}-\bar{X}_{T} \in\left(\sqrt{\frac{2 q b^{2}}{n(n-2)}+\frac{2 q a^{2}}{n(n-2)} U^{2}-4 \frac{\rho a b q}{n(n-2)}} U, \sqrt{Q^{2} S^{2} \frac{1}{n(n-1)}}\right)\right) \\
& =\Phi\left(\frac{\sqrt{Q^{2} S^{2}(1 / n(n-1))}-\left(L \bar{X}_{R}-\bar{X}_{T}\right)}{\sqrt{\left(1+L^{2}\right) \sigma^{2} / n}}\right) \\
& -\Phi\left(\frac{\sqrt{2 q b^{2} / n(n-2)+\left(2 q a^{2} / n(n-2)\right) L^{2}-4(\rho a b q / n(n-2)) L}-\left(L \bar{X}_{R}-\bar{X}_{T}\right)}{\sqrt{\left(1+L^{2}\right) \sigma^{2} / n}}\right) \\
& +\Phi\left(\frac{\sqrt{Q^{2} S^{2}(1 / n(n-1))}-\left(U \bar{X}_{R}-\bar{X}_{T}\right)}{\sqrt{\left(1+U^{2}\right) \sigma^{2} / n}}\right) \\
& -\Phi\left(\frac{\sqrt{2 q b^{2} / n(n-2)+\left(2 q a^{2} / n(n-2)\right) U^{2}-4(\rho a b q / n(n-2)) U}-\left(U \bar{X}_{R}-\bar{X}_{T}\right)}{\sqrt{\left(1+U^{2}\right) \sigma^{2} / n}}\right)=0.10905 \text {, }
\end{aligned}
$$

where $\Phi$ is the cumulative distribution function for standard normal distribution. So $P_{2 U L}<0.10905$, which means the possibility of consistency $P_{2}$ should be greater than $89.095 \%$. Similarly, we could calculate that $P_{1}$ is $100 \%$. 
TABLE 1: Data of the crossover trial.

\begin{tabular}{|c|c|c|c|c|c|c|}
\hline \multirow{2}{*}{ Sequence } & \multirow{2}{*}{ Subject number } & \multicolumn{2}{|c|}{ Period } & \multirow{2}{*}{ Subject total } & \multirow{2}{*}{ P.D. } & \multirow{2}{*}{ C.D. } \\
\hline & & I & II & & & \\
\hline \multicolumn{7}{|l|}{1} \\
\hline $\mathrm{RT}$ & 1 & 74.675 & 73.675 & 148.350 & -1.000 & -1.000 \\
\hline RT & 4 & 96.400 & 93.250 & 189.650 & -3.150 & -3.150 \\
\hline $\mathrm{RT}$ & 5 & 101.950 & 102.125 & 204.075 & 0.175 & 0.175 \\
\hline $\mathrm{RT}$ & 6 & 79.050 & 69.450 & 148.500 & -9.600 & -9.600 \\
\hline RT & 11 & 79.050 & 69.025 & 148.075 & -10.025 & -10.025 \\
\hline $\mathrm{RT}$ & 12 & 85.950 & 68.700 & 154.650 & -17.250 & -17.250 \\
\hline $\mathrm{RT}$ & 15 & 69.725 & 59.425 & 129.150 & -10.300 & -10.300 \\
\hline $\mathrm{RT}$ & 16 & 86.275 & 76.125 & 162.400 & -10.150 & -10.150 \\
\hline RT & 19 & 112.675 & 114.875 & 227.550 & 2.200 & 2.200 \\
\hline RT & 20 & 99.525 & 116.250 & 215.775 & 16.725 & 16.725 \\
\hline $\mathrm{RT}$ & 23 & 89.425 & 64.175 & 153.600 & -25.250 & -25.250 \\
\hline $\mathrm{RT}$ & 24 & 55.175 & 74.575 & 129.750 & 19.400 & 19.400 \\
\hline \multicolumn{7}{|l|}{2} \\
\hline TR & 2 & 74.825 & 37.350 & 112.175 & -37.475 & 37.475 \\
\hline $\mathrm{TR}$ & 3 & 86.875 & 51.925 & 138.800 & -34.950 & 34.950 \\
\hline TR & 7 & 81.675 & 72.175 & 153.850 & -9.500 & 9.500 \\
\hline TR & 8 & 92.700 & 77.500 & 170.200 & -15.200 & 15.200 \\
\hline TR & 9 & 50.450 & 71.875 & 122.325 & 21.425 & -21.425 \\
\hline TR & 10 & 66.125 & 94.025 & 160.150 & 27.900 & -27.900 \\
\hline TR & 13 & 122.450 & 124.970 & 247.420 & 2.525 & -2.525 \\
\hline TR & 14 & 99.075 & 85.225 & 184.300 & -13.850 & 13.850 \\
\hline TR & 17 & 86.350 & 95.925 & 182.275 & 9.575 & -9.575 \\
\hline TR & 18 & 49.925 & 67.100 & 117.025 & 17.175 & -17.175 \\
\hline TR & 21 & 42.700 & 59.425 & 102.125 & 16.725 & -16.725 \\
\hline TR & 22 & 91.725 & 114.050 & 205.775 & 22.325 & -22.325 \\
\hline
\end{tabular}

P.D. $=2 \times($ period difference $)$.

C.D. $=2 \times($ crossover difference $)$.

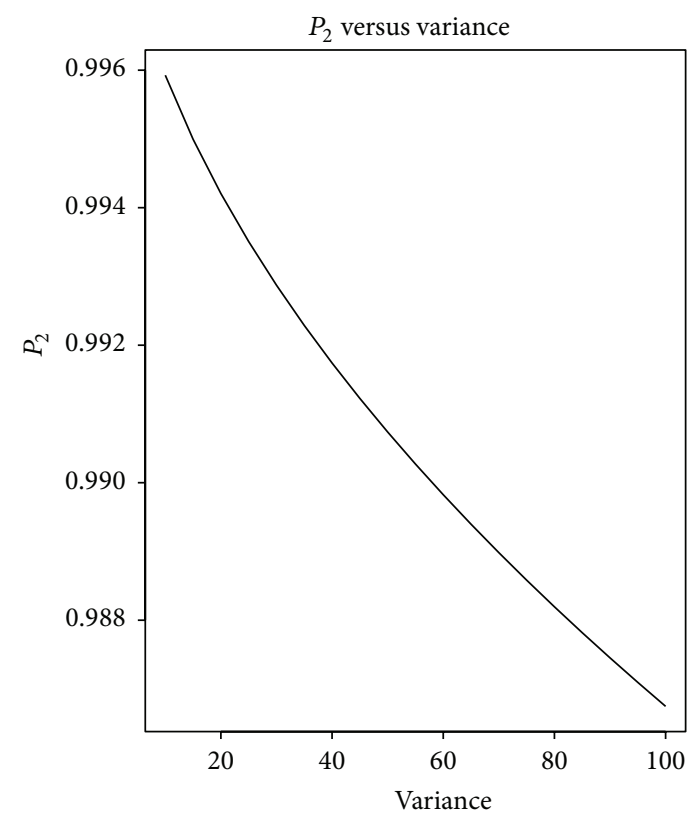

Figure 6: $P_{2}$ against variance based on log-transformed data.

\section{Concluding Remarks}

The traditional one-parameter approach is convenient in examining bioequivalence and could obtain rather accurate results when applying to log-transformed data for generic drugs. However, when applying to raw data, this method lacks accuracy as it assumes the average sample response of the reference products equals $\mu_{R}$. This is especially the case when testing biosimilar products whose response value varies dramatically in different environments. Thus the constructed confidence interval is not the exact confidence interval desired. Besides, tests for biosimilar products may focus on the form $\mu_{T}=a \mu_{R}+b$, where the intercept is not zero. It is hard for one-parameter method to find good test statistics, but the two-parameter method shows its privilege. Meanwhile, the test statistic of the one-parameter approach requires homogeneity, which may only be true for generic drugs. It is not so convincing to overlook the influence of the variation difference when testing biosimilar products.

Our proposed method handles the problems mentioned above. Besides, the two-parameter method can be generalized to higher dimensions circumstances, that is, multiple test 
products and multiple reference products tested at the same time, that is, $\left(\mu_{T 1}, \ldots, \mu_{T m}, \mu_{R 1}, \ldots, \mu_{R m}\right)$. The procedure is similarly finding confidence region and calculates the slope of the hyperplane $\mu_{T i}=k \mu_{R i}(i=1,2, \ldots, m)$. Our proposed method can be applied to all kinds of biosimilar products with only a change of parameter.

As for study design, both parallel and crossover design are viable. However, when performing parallel design, the sample sizes for both formations should be the same. The choice of study design mainly depends on intersubject and intrasubject variance. If intersubject variance is higher, it is more likely to choose crossover design. And parallel design should be priority if it is the other way around. Besides, possibility of ruling out carryover effects and sequence effects should also be taken into consideration.

There are also problems for the two-parameter approach. The approach may not give reasonable result when the origin is within the confidence region. That is,

$$
\frac{\bar{X}_{T}^{2}}{q b^{2}}+\frac{\bar{X}_{R}^{2}}{q a^{2}}-2 \rho \frac{\bar{X}_{R} \bar{X}_{T}}{q a b} \leq \frac{2\left(1-\rho^{2}\right)}{n(n-2)} .
$$

In this situation lines that pass through the origin always intercept the ellipse, so bioequivalence can never be claimed with the two-parameter approach using raw data. For logtransformed data, such limitation does not exist because we can adjust the intercept to find the tangent lines.

Another problem of the two-parameter approach is the restriction on sample size. In times when it is more reasonable to choose parallel design, calculation on sample size could be a new obstacle. The determination of sample size is then important. Methods prevailing nowadays all focus on the one-parameter approach. If performing crossover design, then the methods for one-parameter approach are also feasible. But when conducting parallel design, it is harder to determine. One approach may be requiring $n_{T}=n_{R}$ at the beginning, and then $Y / \sqrt{2 S^{2} / n}$ follows $t$ distribution. Thus we could determine sample size based on statistical power.

The two-parameter approach shows its advantage when applying to biosimilarity test for biosimilar products, because $\mu_{T}=a \mu_{R}+b$ is often required. The intercept $b$ is usually a nonzero parameter, usually between $5 \%$ and $10 \%$, thus providing difficulty for the one-parameter approach. But the criteria for biosimilarity can be easily derived in the twoparameter approach.

In our calculation for consistency, our proposed approach is more rigorous than the one-parameter approach when the sample covariance coefficient is relatively small. Besides, both $P_{1}$ and $P_{2}$ are nonincreasing functions of variance. That is, the two methods are more likely to be divergent when the variances are high. This is conceivable, because the oneparameter method using raw data needs to suppose $\bar{X}_{T}=\mu_{T}$. This assumption is more likely to be false when variances increase. When the test product has way larger variance than the reference products, that is, ${\sigma_{T}}^{2} \gg{\sigma_{R}}^{2}$, we proved that the two-parameter approach will be a more rigorous method. Nevertheless, as the homogeneity assumption does not hold in this situation, we advise the two-parameter approach in assessing biosimilarity.

\section{Appendix}

\section{Calculation of $k$}

The calculation process of deriving the tangent $k$ is shown below. We will only calculate a most general case as is shown in Section 3 Scenario 3, while other results could be obtained by swapping the symbols.

Suppose $A=\left(\begin{array}{cc}a^{2} & \rho a b \\ \rho a b & b^{2}\end{array}\right)$ and the confidence region is of the form

$$
(\bar{X}-\mu)^{\prime} A^{-1}(\bar{X}-\mu) \leq c .
$$

As $A^{-1}=\left(1 / a^{2} b^{2}\left(1-\rho^{2}\right)\right)\left(\begin{array}{cc}b^{2} & -\rho a b \\ -\rho a b & a^{2}\end{array}\right)$, the ellipse function above could be transformed as

$$
\begin{aligned}
& \frac{\left(\mu_{T}-\bar{X}_{T}\right)^{2}}{b^{2}}+\frac{\left(\mu_{R}-\bar{X}_{R}\right)^{2}}{a^{2}} \\
& -2 \rho \frac{\left(\mu_{R}-\bar{X}_{R}\right)\left(\mu_{T}-\bar{X}_{T}\right)}{a b} \leq\left(1-\rho^{2}\right) c .
\end{aligned}
$$

If line $\mu_{T}=k \mu_{R}$ is tangent to the ellipse, then we could get a quadratic function of $\mu_{R}$ :

$$
\begin{aligned}
\left(\frac{k^{2}}{b^{2}}\right. & \left.+\frac{1}{a^{2}}-\frac{2 \rho k}{a b}\right) \mu_{R}^{2} \\
& -2\left(\frac{k \bar{X}_{T}}{b^{2}}+\frac{\bar{X}_{R}}{a^{2}}-\rho \frac{k \bar{X}_{R}+\bar{X}_{T}}{a b}\right) \mu_{R} \\
& +\left(\frac{\bar{X}_{T}^{2}}{b^{2}}+\frac{\bar{X}_{R}^{2}}{a^{2}}-2 \rho \frac{\bar{X}_{T} \bar{X}_{R}}{a b}\right)-\left(1-\rho^{2}\right) c \\
= & 0 .
\end{aligned}
$$

The function's two roots should be the same; then $k$ should satisfy

$$
\begin{aligned}
& 4\left(\frac{k \bar{X}_{T}}{b^{2}}+\frac{\bar{X}_{R}}{a^{2}}-\rho \frac{k \bar{X}_{R}+\bar{X}_{T}}{a b}\right)^{2} \\
& -4\left(\frac{k^{2}}{b^{2}}+\frac{1}{a^{2}}-\frac{2 \rho k}{a b}\right) \\
& \cdot\left(\frac{\bar{X}_{T}^{2}}{b^{2}}+\frac{\bar{X}_{R}^{2}}{a^{2}}-2 \rho \frac{\bar{X}_{T} \bar{X}_{R}}{a b}-\left(1-\rho^{2}\right) c\right)=0
\end{aligned}
$$


that is,

$$
\begin{aligned}
& k^{2}\left(\frac{\bar{X}_{T}^{2}}{b^{4}}+\rho^{2} \frac{\bar{X}_{R}^{2}}{a^{2} b^{2}}-\frac{2 \rho \bar{X}_{T} \bar{X}_{R}}{a b^{3}}-\frac{\bar{X}_{T}^{2}}{b^{4}}-\frac{\bar{X}_{R}^{2}}{a^{2} b^{2}}\right. \\
& \left.+\frac{2 \rho \bar{X}_{R} \bar{X}_{T}}{a b^{3}}+\frac{\left(1-\rho^{2}\right) c}{b^{2}}\right) \\
& +2 k\left(\frac{\bar{X}_{T} \bar{X}_{R}}{a^{2} b^{2}}\left(1+\rho^{2}\right)-\rho \frac{\bar{X}_{T}^{2}}{a b^{3}}-\rho \frac{\bar{X}_{R}^{2}}{a^{3} b}\right. \\
& \left.+\rho \frac{\bar{X}_{T}^{2}}{a b^{3}}+\rho \frac{\bar{X}_{R}^{2}}{a^{3} b}-2 \rho^{2} \frac{\bar{X}_{T} \bar{X}_{R}}{a^{2} b^{2}}-\frac{\rho\left(1-\rho^{2}\right) c}{a b}\right) \\
& +\left(\frac{\bar{X}_{R}}{a^{2}}-\rho \frac{\bar{X}_{T}}{a b}\right)^{2}-\frac{1}{a^{2}}\left(\frac{\bar{X}_{T}^{2}}{b^{2}}+\frac{\bar{X}_{R}^{2}}{a^{2}}\right. \\
& \left.+2 \rho \frac{\bar{X}_{T}}{a b}-\left(1-\bar{X}_{R}^{2}\right) c\right)=0 ;
\end{aligned}
$$

thus,

$$
\begin{aligned}
& k^{2}\left[\left(\rho^{2}-1\right) \frac{\bar{X}_{R}^{2}}{a^{2} b^{2}}+\frac{\left.\left(1-\rho^{2}\right) c\right]}{b^{2}}\right] \\
& +2 k\left[\left(-\rho^{2}+1\right) \frac{\bar{X}_{T} \bar{X}_{R}}{a^{2} b^{2}}-\frac{\rho\left(1-\rho^{2}\right) c}{a b}\right] \\
& +\frac{\bar{X}_{T}^{2}}{a^{2} b^{2}}\left(\rho^{2}-1\right)+\frac{\left(1-\rho^{2}\right) c}{a^{2}}=0, \\
& k^{2}\left(\frac{c}{b^{2}}-\frac{\bar{X}_{R}^{2}}{a^{2} b^{2}}\right)+2 k\left(\frac{\bar{X}_{T} \bar{X}_{R}}{a^{2} b^{2}}-\frac{\rho c}{a b}\right)+\frac{c}{a^{2}} \\
& \quad-\frac{\bar{X}_{T}^{2}}{a^{2} b^{2}}=0, \\
& k^{2}\left(\bar{X}_{R}^{2}-a^{2} c\right)-2 k\left(\bar{X}_{T} \bar{X}_{R}-\rho a b c\right) \\
& +\left(\bar{X}_{T}^{2}-b^{2} c\right)=0 .
\end{aligned}
$$

Then we could calculate the slope of the tangent lines:

$$
\begin{aligned}
\Delta \triangleq & {\left[-\bar{X}_{T} \bar{X}_{R}+\rho a b c\right]^{2} } \\
& -\left(\bar{X}_{R}^{2}-c a^{2}\right)\left(\bar{X}_{T}^{2}-c a^{2}\right), \\
k= & \frac{\bar{X}_{T} \bar{X}_{R}-\rho a b c \pm \sqrt{\Delta}}{\left(\bar{X}_{R}^{2}-a^{2} c\right)} .
\end{aligned}
$$

$\mu_{T}=U \mu_{R}$ and $\mu_{T}=L \mu_{R}$ should not intersect with the ellipse, so when $k$ is substituted by $U$ or $L$ in the discrimination function above, we should get

$$
\begin{aligned}
& 4\left(\frac{K \bar{X}_{T}}{b^{2}}+\frac{\bar{X}_{R}}{a^{2}}-\rho \frac{K \bar{X}_{R}+\bar{X}_{T}}{a b}\right)^{2} \\
& \quad-4\left(\frac{K^{2}}{b^{2}}+\frac{1}{a^{2}}-\frac{2 \rho K}{a b}\right) \\
& \quad\left(\frac{\bar{X}_{T}^{2}}{b^{2}}+\frac{\bar{X}_{R}^{2}}{a^{2}}-2 \rho \frac{\bar{X}_{T} \bar{X}_{R}}{a b}-\left(1-\rho^{2}\right) c\right)<0, \\
& K^{2}\left(\bar{X}_{R}^{2}-a^{2} c\right)-2 K\left(\bar{X}_{T} \bar{X}_{R}-\rho a b c\right) \\
& \quad+\left(\bar{X}_{T}^{2}-a^{2} c\right)>0 ;
\end{aligned}
$$

that is,

$$
\left(K \bar{X}_{R}-\bar{X}_{T}\right)^{2}>a^{2} c+a^{2} c K^{2}-2 K \rho a b c,
$$

where $K=U$ or $L$.

\section{Conflict of Interests}

The authors declare that there is no conflict of interests regarding the publication of this paper.

\section{References}

[1] S. C. Chow and J. Shao, "An alternative approach for the assessment of bioequivalence between two formulations of a drug," Biometrical Journal, vol. 32, no. 8, pp. 969-976, 1990.

[2] H.-C. Hsu and H.-L. Lu, "On confidence limits associated with Chow and Shao's joint confidence region approach for assessment of bioequivalence," Journal of Biopharmaceutical Statistics, vol. 7, no. 1, pp. 125-134, 1997.

[3] FDA, Guidance on Bioavailability and Bioequivalence Studies for Orally Administrated Drug Products-General Considerations, Center for Drug Evaluation and Research, U.S. Food and Drug Administration, Rockville, Md, USA, 2003.

[4] S. C. Chow and S. K. Tse, "A related problem in bioavailability/bioequivalence studies-estimation of the intrasubject variability with a common CV,' Biometrical Journal, vol. 32, no. 5, pp. 597-607, 1990.

[5] Y. Lee, J. Shao, S.-C. Chow, and H. Wang, "Tests for intersubject and total variabilities under crossover designs," Journal of Biopharmaceutical Statistics, vol. 12, no. 4, pp. 503-534, 2002.

[6] W. A. Morgan, "Test for the significance of the difference between the two variances in a sample from a normal bivariate population," Biometrika, vol. 31, no. 1-2, pp. 13-19, 1939.

[7] E. J. G. Pitman, "A note on normal correlation," Biometrika, vol. 31, pp. 1-12, 1939.

[8] S. C. Chow and J. P. Liu, Design and Analysis of Bioavailability and Bioequivalence Studies, Chapman \& Hall, CRC Press, Taylor \& Francis, New York, NY, USA, 3rd edition, 2009. 


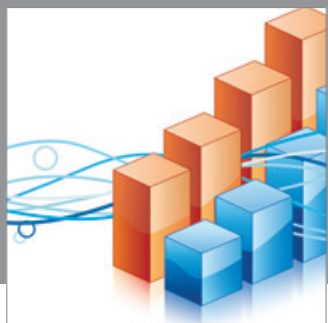

Advances in

Operations Research

mansans

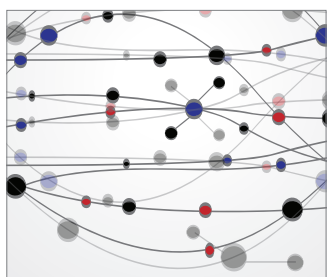

The Scientific World Journal
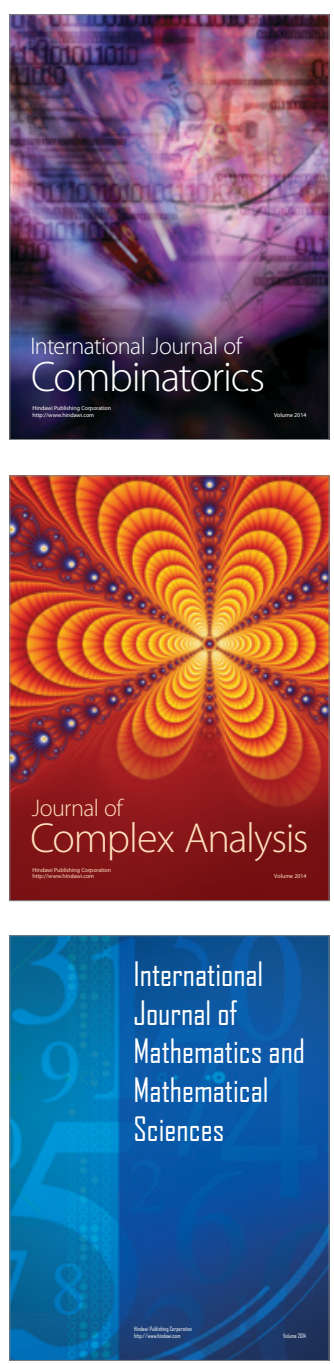
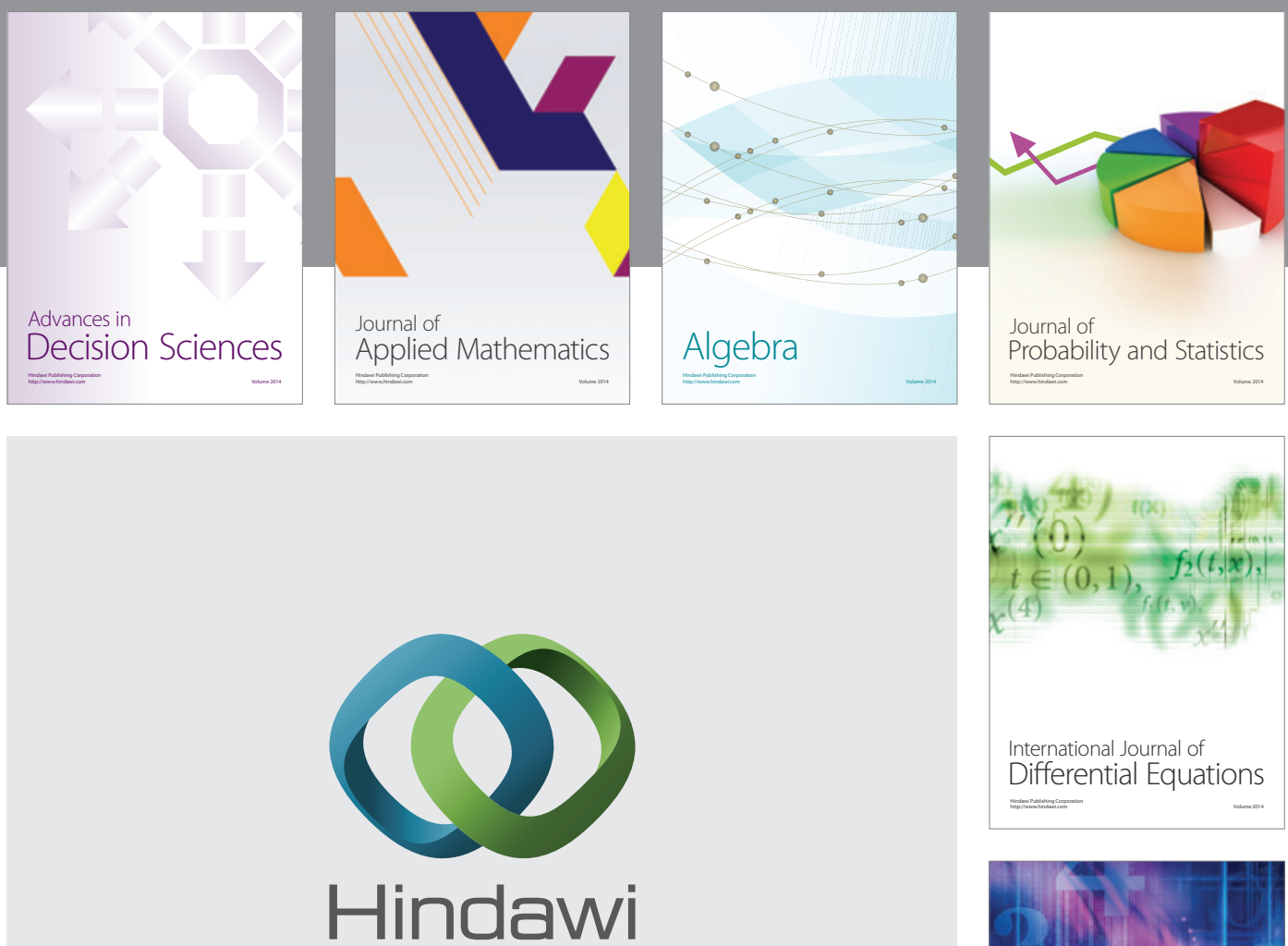

Submit your manuscripts at http://www.hindawi.com
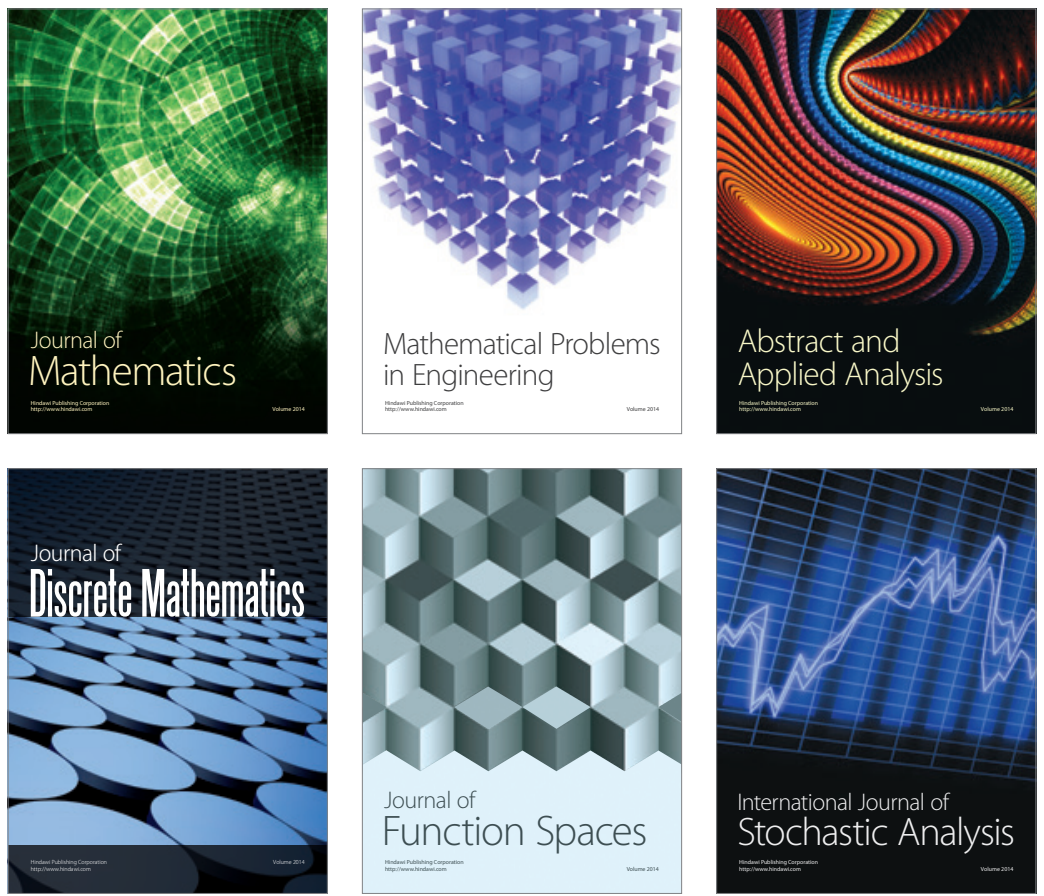

Journal of

Function Spaces

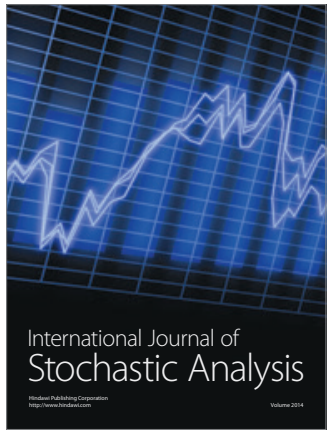

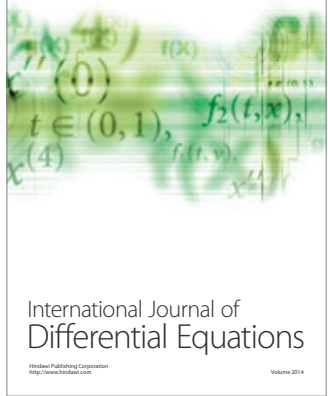
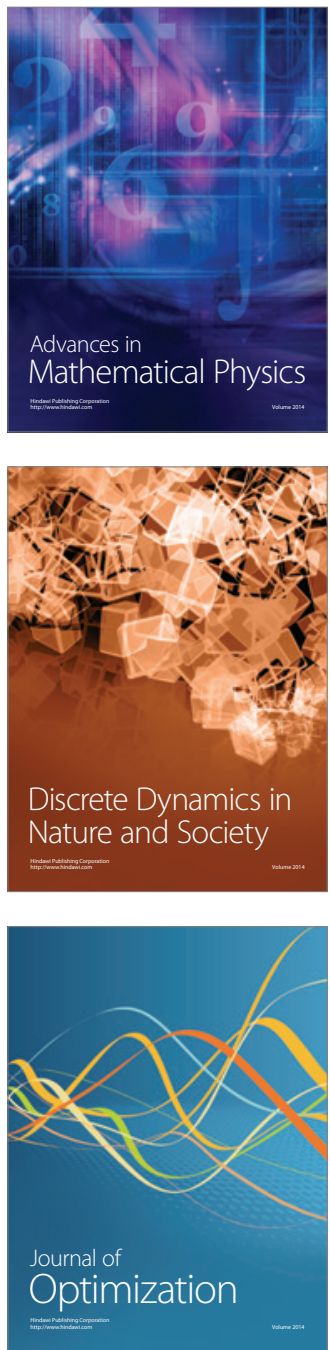